\title{
The Computer-Assisted Cognitive/Imagery System for use in the management of pain
}

\author{
Jeffrey J Borckardt $\mathrm{PhD}^{1}$, Jarred Younger $\mathrm{PhD}^{2}$, Justin Winkel $\mathrm{BA}^{3}$, Michael R Nash $\mathrm{PhD}^{3}$, Darlene Shaw $\mathrm{PhD}^{1}$
}

\begin{abstract}
JJ Borckardt, J Younger, J Winkel, MR Nash, D Shaw. The Computer-Assisted Cognitive/Imagery System for use in the management of pain. Pain Res Manage 2004;9(3):157-162.
\end{abstract}

BACKGROUND: There is growing interest in computer-delivered psychological interventions for a number of clinical conditions, including pain.

OBJECTIVES: This study tests the effectiveness of a new computerdelivered pain-management program using a laboratory pain paradigm. METHODS: One hundred twenty undergraduate students were randomly assigned to either the computerized pain-management group or the distraction control group. Subjects underwent a cold-pressor task and were asked to continuously rate their subjective pain experience. RESULTS: Women receiving the computerized pain management intervention were able to tolerate the cold-pressor task longer than those in the control group. No effect was found for men. Subjective pain ratings were consistently lower during the cold-pressor task for subjects in the computerized pain-management group regardless of sex. Subjects receiving the computerized intervention reported feeling more comfortable and relaxed than control subjects during the cold-pressor task.

CONCLUSIONS: Findings indicate that further investigations of the program used in this study are warranted to determine its potential clinical utility and that of similar computerized interventions for pain.

Key Words: Cognitive; Computer; Hypnosis; Imagery; Pain

\section{Programme d'imagerie et de thérapie cognitive assistées par ordinateur pour le soulagement de la douleur}

CONTEXTE : Les interventions psychologiques assistées par ordinateur suscitent un intérêt croissant pour le traitement de certains troubles cliniques, notamment de la douleur.

BUT : La présente étude avait pour but de vérifier l'efficacité d'un nouveau programme de soulagement de la douleur, assisté par ordinateur à l'aide d'un protocole d'étude de la douleur en laboratoire.

MÉTHODE : Cent vingt étudiants de premier cycle ont été hasardisés vers le groupe de soulagement de la douleur, assisté par ordinateur ou vers le groupe témoin ayant recours à la diversion. Les sujets ont été soumis à une épreuve au froid et on leur a demandé de coter en continu leur degré subjectif de douleur.

RÉSULTATS : Les femmes soumises à l'intervention assistée par ordinateur ont été capables de tolérer l'épreuve plus longtemps que celles dans le groupe témoin. Par contre, les hommes se sont montrés insensibles à l'intervention. Par ailleurs, les cotes subjectives de la douleur ont été plus basses dans le groupe de soulagement de la douleur, assisté par ordinateur que dans l'autre, et ce, tout au long de l'épreuve, sans distinction de sexe. Les sujets du groupe expérimental se sont dits plus détendus et plus à l'aise que les sujets du groupe témoin durant l'épreuve.

CONCLUSION : Les résultats indiquent qu'une évaluation plus poussée du programme utilisé dans l'étude serait justifiée pour déterminer ses possibilités d'application clinique, de même que celles d'autres interventions similaires, assistées par ordinateur.
$\mathrm{T}$ here is a growing trend toward the integration of computer technology into psychological services. Computers have been used extensively for psychological test scoring, printing and psychological report generation, but the field of psychology seems to be moving more toward the use of computers in training $(1,2)$ and clinical service delivery (3). This movement is in part driven by a need to provide better access to psychological services in primary care and underserved populations as well as to cut perpatient costs of cognitive-behavioural therapy without sacrificing clinical effectiveness (4). Computerized psychotherapy and psychoeducation modules continue to be researched and implemented in the treatment of various psychological problems, including simple phobias, panic disorder, depressive disorders, social anxiety, post-traumatic stress disorder, eating disorders and sexual dysfunction (5-10). Additionally, there is a recent trend toward the use of hand-held (palmtop) computers as adjunctive tools to psychotherapy for generalized anxiety disorder, social phobia and panic disorder (11-13).
While many early computer psychotherapy modules were largely didactic in nature and focused on psychoeducation interventions, several programs have been developed to be more interactive $(8,14)$. The use of the internet has furthered this aim by providing psychotherapy modules that sometimes involve real human interactions carried out in cyberspace (15-18).

Another new technology that has been widely applied with regard to psychological services is virtual reality (VR). VR involves immersing patients in an interactive computerized threedimensional world that can be modified to contain specific elements that may be relevant to certain psychological/behavioural problems and/or clinical goals. VR has been used to deliver exposure-based therapies for various phobias, including fear of heights, flying, claustrophobia, driving and spiders (5).

There has been considerable research conducted on the use of VR in the management of pain (19-21). This is an important line of research because chronic pain conditions are poorly

\footnotetext{
${ }^{1}$ Medical University of South Carolina, Charleston, South Carolina; ${ }^{2}$ University of Arizona, Tucson, Arizona; ${ }^{3}$ University of Tennessee, Knoxville, Tennessee, USA

Correspondence: Dr Jeffrey J Borckardt, 30 Bee Street (CAPS), Medical University of South Carolina, Department of Psychiatry and Behavioral Sciences, Charleston, South Carolina, USA 29425. Telephone 843-792-4930, fax 843-792-2535, e-mail borckard@musc.edu
} 


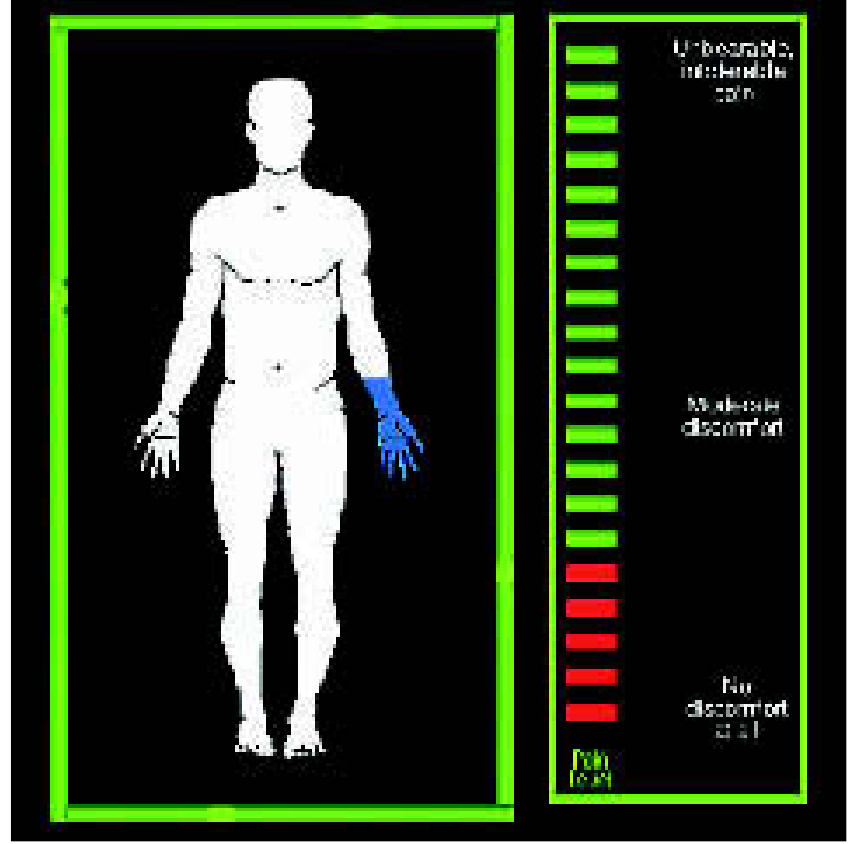

Figure 1) The main screen of the computer-assisted imagery program with the male figure image. The subjective pain meter is at the right and is controlled by keys on the computer keyboard. The blue area on the figure corresponds to the portion of the subject's arm immersed in the circulating ice bath

understood, poorly treated, and are a significant public health concern costing billions of dollars each year in lost wages, lost productivity and disability expenses (22). Additionally, acute pain conditions can be problematic to manage and risky because use of anesthesia (general and local) during some medical procedures can sometimes be contraindicated and often does not provide adequate pain relief. There is a growing body of evidence that VR can be used to significantly reduce pain in laboratory paradigms and the clinic (eg, burn débridement, dental procedures). VR is generally believed to work for the treatment of pain by distracting subjects from the noxious stimuli responsible for the pain $(18,19)$.

Several psychological interventions and procedures have been shown to be effective in the management of acute and chronic pain conditions. Specifically, cognitive-behavioural therapy, group therapy, relaxation training and psycho-educational interventions have been shown to reduce pain intensity and increase functioning in chronic pain sufferers $(23,24)$. There is also extensive empirical support for the use of hypnosis in the management of chronic and acute pain conditions (25-27). These effects appear to exceed placebo and/or relaxation effects and seem to be dependent on the patient's natural abilities to respond to hypnotic suggestions for analgesia, dissociation or distraction rather than the hypnotist's authority, demeanor, voice or wording of suggestions.

Grant and Nash (28) introduced a computer-delivered hypnotic assessment program that was shown to have acceptable reliability and validity. The program administered a hypnotic induction and then assessed responsiveness to suggestion using behavioural items that were scored either by the subject or the computer depending on the item. This project demonstrated that the computer is a feasible medium for delivering hypnotic procedures.
While computers have been used to manage various clinical pain conditions and administer hypnotic procedures, there have not been many computer programs developed to deliver hypnotically-based interventions for pain. This is unfortunate given the strong empirical evidence demonstrating its clinical efficacy for acute and chronic pain conditions.

The present study seeks to test the effectiveness of a computer-administered pain management program using normal college student subjects in a laboratory pain paradigm. The program was based on widely accepted hypnotic and cognitive procedures for pain management to help subjects better tolerate experimental pain. It was hypothesized that subjects receiving the computer-delivered pain management program would be able to tolerate a cold-pressor pain task longer than subjects who were asked to listen to a story (serving as a mild distraction control condition). Additionally, subjective pain ratings were hypothesized to be lower in the pain program group than in the control group. Lastly, it was hypothesized that subjects receiving the pain program would report that they felt more comfortable and relaxed during the cold-pressor task than control subjects.

\section{METHODS}

\section{Subjects}

One hundred twenty undergraduate students enrolled in psychology courses at the University of Tennessee were given extra credit for participation in this study. There were 52 men and 68 women with a mean age of 20.41 . There were 26 men and 38 women in the control group, and 26 men and 30 women in the experimental group ( $\chi^{2}$ [degrees of freedom $\left.=1, n=120\right]=0.41$, not significant). The mean age of subjects in the control group was 20.29 $(\mathrm{SD}=2.38)$ and the mean age of subjects in the experimental group was $20.52(\mathrm{SD}=2.86)$. No age-differences were found for sex, group-assignment, or the sex by group interaction $(F[3,116]=0.84$, not significant $)$. It appears that the randomization procedure did not result in any systematic bias with respect to the age and sex of subjects assigned to each group.

\section{Computer-Assisted Cognitive/Imagery System}

The Computer-Assisted Cognitive/Imagery System (CACIS) was developed using an object-oriented visual basic environment for Mac Os (RealBasic 4.02) (Apple Computer Inc, USA) and crosscompiled for Windows95/98/NT (Microsoft Corporation, USA) by the first author. It was administered using the Windows 98 platform on a Dell Computer (Dell Inc, USA) (300 MHz Processor and $64 \mathrm{MB}$ of RAM).

On starting the program, users are prompted for their age and sex. The main program interface depicts a blank human figure on a black screen. The sex of the figure automatically adjusts to match the indicated sex of the subject. The portion of the human figure that corresponds with the location of the subject's pain is highlighted with colour. For the present study, the left hand and forearm of the figure was coloured blue to correspond with the location of sensations experienced during the coldpressor task.

There is a digital visual pain meter to the right of the figure that is controlled with the 'arrow' keys on the keyboard. The scale ranges from one to 20 , although the numbers are not visible on the screen. The scale has three text anchors: "no discomfort at all," "moderate discomfort" and "unbearable, intolerable pain." Figure 1 shows a screenshot of the main interface. 
The program begins by playing a 4 min recording of a male voice providing a brief hypnotic induction comprised of verbal instructions to relax, breath freely and deeply, and focus attention on the computer screen. Suggestions are also given to attend to bodily sensations (eg, the feeling of the chair against the subject's body) as a means of demonstrating to the subject that they can choose which sensations to attend to at any given time. Efforts are made to cognitively frame the pain experience for the subject so that it is 'uncomfortable' rather than 'painful' and in such a way that emphasis is placed on the notion that the subject's experience is under his/her control. During this introduction, the green frame around the human figure appears to slowly rotate around the figure to be more visually captivating.

The CACIS program has three modules that were all implemented in the same order for the present study. Throughout all the modules, the pain meter was controlled by subjects using the arrow keys. The level of the meter was silently recorded by the program every $2 \mathrm{~s}$ and the values were saved to an SPSS database (SPSS Inc, USA).

Module 1 involves suggestions to imagine the sensations (of cold-pressor pain) as the colour blue, corresponding with the human figure on the screen. Suggestions are then given to imagine that the colour is slowly changing to a 'warmer' colour. As these suggestions are given, the program animates the human figure such that the blue colour (representing cold-pressor pain) in the left forearm slowly changes to red.

Module 2 involves suggestions for imagining the sensations of pain 'fading away' and 'disintegrating.' While these suggestions are being given, the image of the pain sensation on the screen (now red) slowly disintegrates and fades to white.

Module 3 involves suggestions for any residual pain sensations to 'leave the body' and drift away. While these suggestions are given, the image on the screen animates such that the residual red dots representing pain in the figures arm float out of the arm and off the screen.

Figure 2 shows progressive screen shots of images from each of the three modules.

\section{Control-group program}

The control-group program is identical to the CACIS program in terms of computer screen layout and operation of the digital pain meter. However, instead of the script used in the CACIS program, the computer plays a recording of the same male voice reading a story about bird migratory patterns (matched for length). Additionally, the control program presents the human figure with the blue colouring on the left forearm; however, no animation is used and no suggestions are given for altered experiences or visual imagery. The subject is simply instructed to relax and listen carefully to a short story.

\section{Cold-pressor task}

A $75.71 \mathrm{~L}$ insulated plastic rectangular tank was filled with ice water and an electric pump was attached inside the container to constantly circulate the water. A digital thermometer was mounted inside the tank and water temperature was monitored and maintained at $0.56^{\circ} \mathrm{C}$ by adding ice when necessary.

Subjects submerged their left hand and arm in the ice bath up to the elbow. They were instructed to remove their arm from the ice bath when they could not tolerate the discomfort any longer. Subjects were not allowed to keep their arms in the ice bath for longer than $150 \mathrm{~s}$. Subjects were given a warm, dry towel after removing their arms from the ice bath.
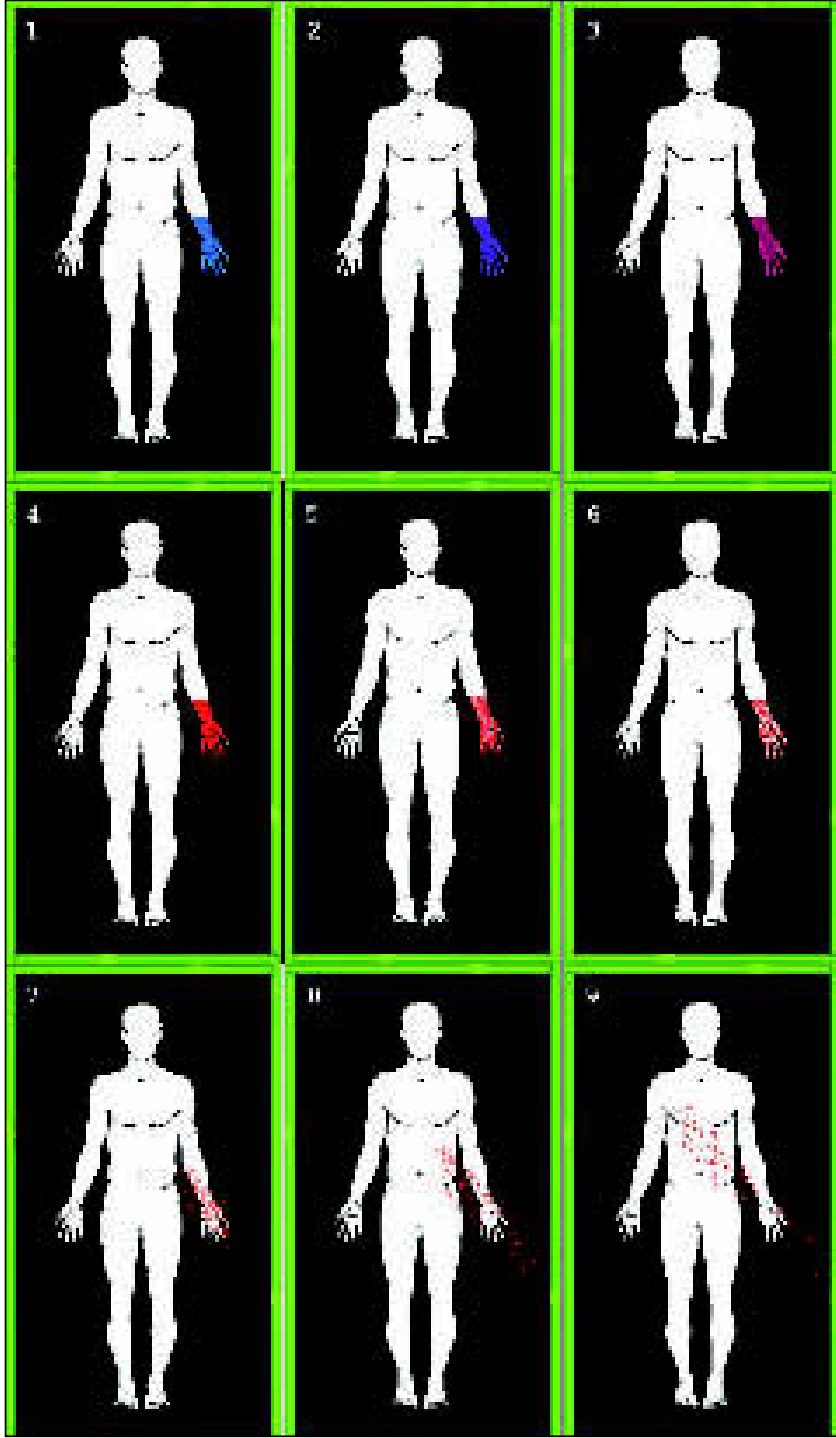

Figure 2) Still images of various frames throughout the computerassisted imagery animation program showing the "warming" phase (frames 1-4), the "fading" phase (frames 5-6) and the "drifting-away" phase (frames 7-9)

\section{Follow-up questions}

After completing the task, subjects were prompted by the computer to indicate how comfortable they felt during the cold-pressor task and how relaxed they felt during the cold-pressor task using a 5-point Likert scale (1=not at all, 5=completely).

\section{Procedure}

Subjects were brought individually to a sound-proof laboratory room in the psychology building at the University of Tennessee. They read and signed the informed consent form and were given an opportunity to ask questions about the cold-pressor task. Subjects were told that they would be doing the cold-pressor task while interacting with a computer but subjects were not made aware of the aims, design or hypotheses of the study. Subjects sat at the computer and completed a $1 \mathrm{~min}$ practice trial using the digital pain meter to get familiar with the scale and the controls with the help of a lab assistant. Subjects then started the program and the research assistant moved out of the subject's line of sight. Once started, the program 


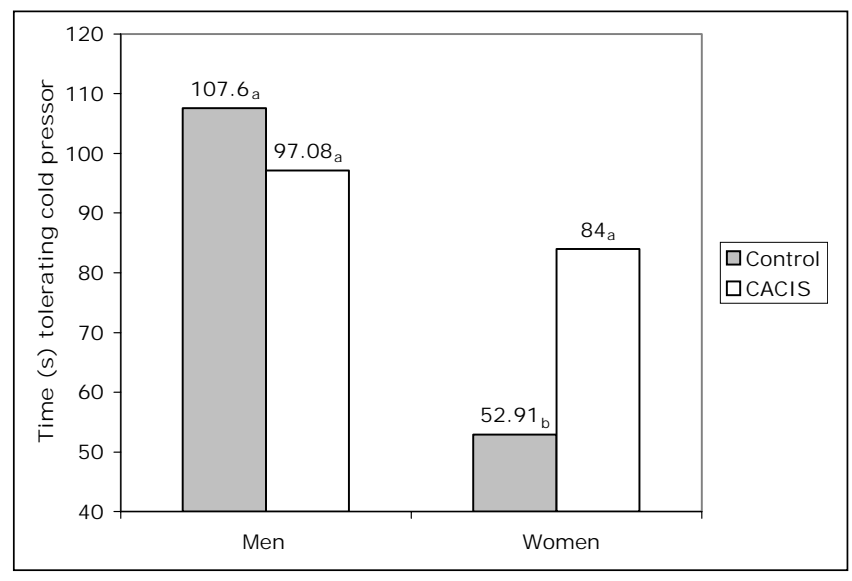

Figure 3) Mean time in seconds that men and women in the Computer-Assisted Cognitive/Imagery System (CASIS) group and the control group tolerated the cold-pressor task (different subscripts indicate significant differences)

silently (randomly) assigned the subject to either the CACIS program or the control program and started the program.

After the 4 min introduction (CACIS group) or the first $4 \mathrm{~min}$ of the bird-story (control group), the computer instructed subjects to place their left arm in the ice bath and begin using the digital meter to indicate their level of discomfort. Then, the CACIS program began the modules (CACIS group) or the computer continued the bird-story (control group). Subjects were instructed to remove their arm from the ice bath at any time they could no longer tolerate the discomfort. They were instructed to press the spacebar when they removed their arm from the water to indicate to the computer that they stopped the task. The computer program tracked the length of time each subject kept his/her arm in the ice-bath. The research assistant sat behind the subjects to ensure that the cold-pressor task was started at the right time and to make sure the spacebar was pressed when the subject removed his/her hand from the water. Subjects were given a warm, dry towel and answered the questions about their level of comfort and relaxation during the task. They were thanked for their participation and thoroughly debriefed.

\section{RESULTS}

\section{Cold-pressor tolerance}

Subjects in the control group were able to tolerate the coldpressor task for a mean of $73.25 \mathrm{~s}$, whereas subjects receiving the CACIS program tolerated the cold-pressor for a mean of $86.25 \mathrm{~s}(\mathrm{t}[118]=1.40, \mathrm{P}=0.16)$. While this failed to reach statistical significance, a $2 \times 2$ ANOVA with group (control versus CACIS) and sex (male versus female) indicated a significant main effect for sex $(\mathrm{F}[1,116]=13.40, \mathrm{P}<0.0001)$ and a significant interaction between group and sex regarding the length of time subjects were able to tolerate the cold-pressor pain $(F[1,116]=6.55, P=0.012)$. Figure 3 displays the means for men and women in the control and CACIS groups. Women in the control condition were unable to tolerate the cold-pressor pain as long as women in the CACIS group or as long as men.

Thirty-three subjects (27.5\%) kept their arms in the coldpressor for the entire $150 \mathrm{~s}$ (ceiling effect). There was an approximately equal number of subjects in the control and CACIS group who reached the ceiling (16 and 17, respectively). However, there were twice as many men $(n=22)$ as women

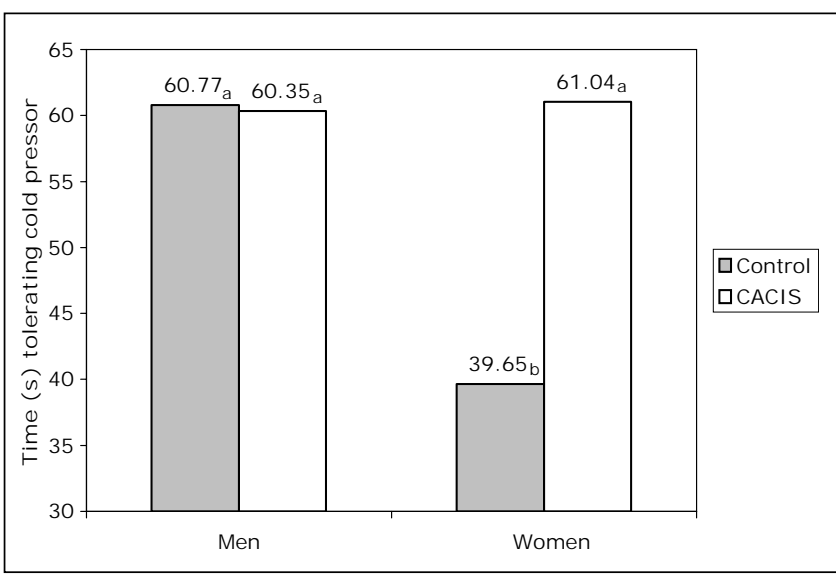

Figure 4) Mean time in seconds that men and women in the Computer-Assisted Cognitive/Imagery System (CASIS) group and the control group tolerated the cold-pressor task after the ceiling effects are removed from the analysis (different subscripts indicate significant differences)

$(n=11)$ that reached the ceiling. Figure 4 shows the mean time that male and female control and CACIS subjects tolerated the cold-pressor after subjects who reached the ceiling were removed from the analyses. It appears that women in the control group had lower pain tolerances than men in the control group. While the CACIS program did not appear to provide any significant benefit to the men in our sample, the CACIS program increased the women's pain tolerance to match that of the men in our sample.

\section{Subjective pain ratings}

Subjective pain ratings (collected silently by the computer program every $2 \mathrm{~s}$ ) were examined throughout the first $60 \mathrm{~s}$ of the cold-pressor task. Since subjects were to remove their arms from the ice bath when they were unable to tolerate the discomfort any longer (and therefore no longer gave pain ratings), the number of subjects contributing to the mean pain rating at any given time-point varied. Specifically, there were fewer and fewer subjects available to give pain ratings across time during the cold-pressor task. By $60 \mathrm{~s}$ into the task, half of the subjects had discontinued and thus mean estimates became much less reliable during later portions of the task. Figure 5 shows the mean subjective pain ratings over time between the control and CACIS groups for the first $60 \mathrm{~s}$ of the cold-pressor task. Ratings from subjects in the CACIS group were consistently lower than ratings from subjects in the control group. Figure 6 shows the mean subjective pain ratings over time between men and women. Men consistently gave lower pain ratings than women throughout the first $60 \mathrm{~s}$ of the cold-pressor task.

\section{Follow-up questions}

Subjects in the CACIS group indicated that they were significantly more comfortable during the cold-pressor task than control subjects $(\mathrm{t}[110]=3.26, \mathrm{P}=0.001)$. Additionally, CACIS subjects reported being more relaxed during the cold-pressor task $(\mathrm{t}[110]=2.27, \mathrm{P}=0.025)$. Figure 7 shows the mean ratings for each group on each of the self-report questions.

\section{DISCUSSION}

Pain, once thought as an inevitable consequence of certain medical procedures and chronic conditions, is a phenomenon 


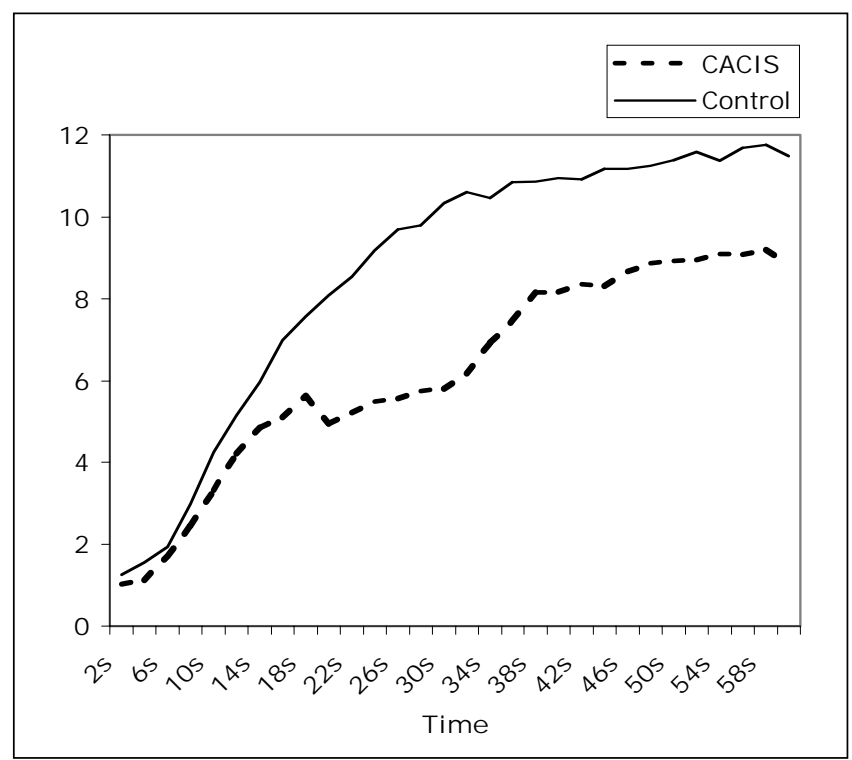

Figure 5) Mean subjective pain ratings from subjects in the ComputerAssisted Cognitive/Imagery System (CACIS) Group and the control group during the first $60 \mathrm{~s}$ of the cold-pressor task recorded every $2 \mathrm{~s}$ by the computer program

receiving greater amounts of attention from the scientific and medical communities. Furthermore, the rising incidence rates of chronic pain conditions such as rheumatoid arthritis, fibromyalgia and irritable bowel syndrome have increased the need for effective techniques for controlling pain.

The present study demonstrates that hypnotic/imagery techniques can be combined with computer-based protocols to offer a pain intervention tool that works for experimentally induced acute pain. The use of the computerized hypnosis/imagery program was superior to the mild distraction program in lowering experienced pain. Both men and women indicated that they were significantly more comfortable and relaxed when using the CACIS program as opposed to distraction during the cold-pressor task. These post-task measures were further supported by pain data collected during the task, which showed the CACIS group experiencing significantly less pain than the control group. Lastly, the effectiveness of the CACIS technique was validated by behavioural responses (eg, the amount of time subjects' hands stayed in the water), although this effect was found for women only. Because men showed no significant difference in the time they withstood the task despite reporting elevated pain in the control condition, we infer that other factors (besides degree of experienced pain) determined how long men chose to endure the painful laboratory stimulus, or that the maximum period of arm exposure was not sufficient enough to reveal individual differences in pain tolerance among men (eg, ceiling effect).

Our laboratory findings document that computer-based hypnotic-cognitive interventions can be effective helping subjects manage experimental pain. This is promising. However, serious questions remain, not the least of which is whether the benefit of this computer-based intervention will generalize from the laboratory to the clinic. Will this intervention be helpful to patients suffering from chronic pain? Will it help patients better tolerate acute instructive pain of some medical procedures? Will it do either of these things as

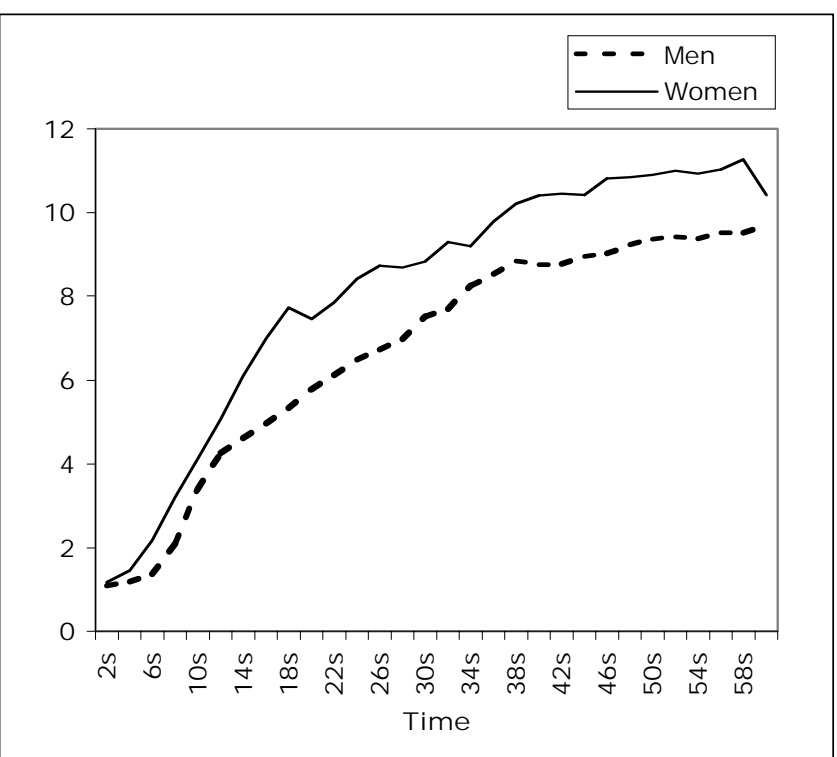

Figure 6) Mean subjective pain ratings from male and female subjects during the first $60 s$ of the cold-pressor task recorded every $2 s$ by the computer program

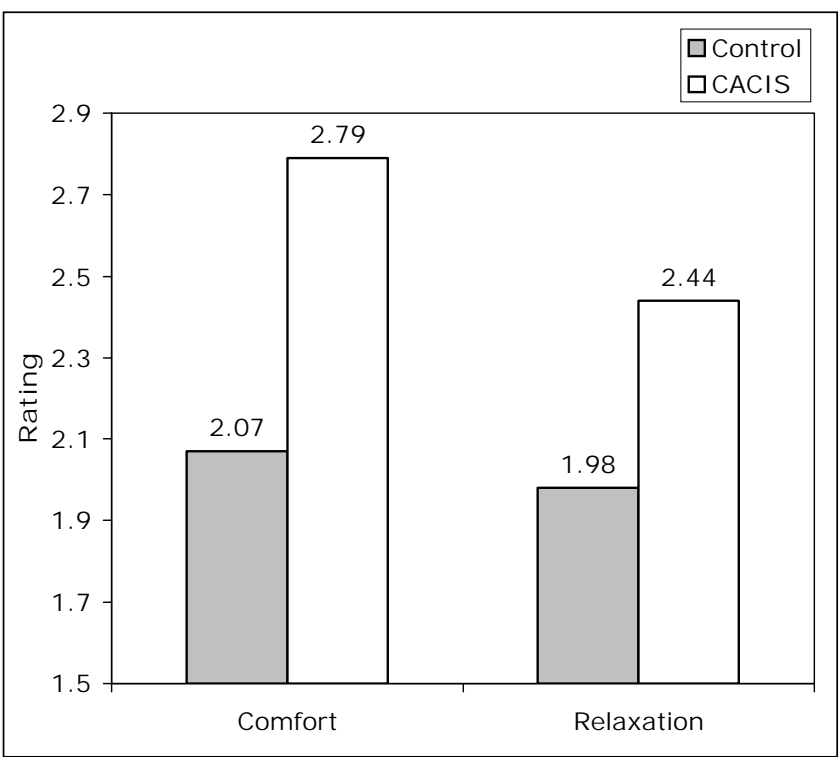

Figure 7) Mean self-report ratings of subjective comfort and overall sense of relaxation during the cold-pressor task for subjects in the Computer-Assisted Cognitive/Imagery System (CACIS) group and the control group. Differences between control and CACIS groups are both significant $(P<0.05)$

well as, or better, than proven distraction techniques presently used in clinics?

Furthermore, little attempt was made in the present study to identify the many factors that might make an individual more or less responsive to the CACIS technique. The only individual variable measured was sex, which did interact with the effectiveness of the program. These results support previous findings showing that men and women react differently to pain-intervention techniques (29) and lend further credence to calls for sex-specific pain management strategies (30). 


\section{CONCLUSIONS}

Based on the information provided by this study, we do not know what specific aspects of the CACIS program were associated with significant benefit (eg, audio, suggestions, visual stimulation/distraction, animation, relaxation effects, etc). Future studies should investigate which aspects of the CACIS program provide the most benefit by systematically removing and/or adding different components of the program and testing the modified program against other versions. Fortunately, because of the nature of computer-delivered interventions, modifications such as these can be implemented quite easily.

Personality and cognitive variables may also determine to what extent an individual reacts to any one pain-management technique. Several distinct pain-response styles have been identified (31) and pain control has been found to be more effective when coping styles and pain management strategies are congruent (32). With regard to the present study, sensitizers may be more responsive to the CACIS protocol while repressors would find distraction techniques to be more helpful. Cognitive factors (such as imagery ability) may further shape the effectiveness of pain management interventions. One of the greatest benefits of computerized pain-control programs is their flexibility. As future studies determine the factors that best predict efficacious pain-management strategies, computers may be able to both assess and deliver the most appropriate program for each individual. These programs may free up time for medical professionals and the portability of newer devices make ambulatory pain-management protocols a feasible option for chronic pain or postoperative patients. The results of the present study provide continuing support for the use of computers in pain intervention.

\section{REFERENCES}

1. Mar CM, Chabal C, Anderson RA, Vore AE. An interactive computer tutorial to teach pain assessment. J Health Psychol 2003;8:161-73.

2. Schultheis MT, Himelstein J, Rizzo AA. Virtual reality and neuropsychology: Upgrading the current tools. J Head Trauma Rehabil 2002;17:378-94.

3. Bornas X, Rodrigo T, Barcelo F, Toledo M. New technologies in cognitive behavioral therapy: A review. Int J Clinical Health Psychol 2002;2:533-41.

4. Marks IM, Mataix-Cols D, Kenwright M, Cameron R, Hirsch S, Gega L. Pragmatic evaluation of computer aided self help for anxiety and depression. Br J Psychiatry 2003;183:57-65.

5. Glanz K, Rizzo A, Graap K. Virtual reality for psychotherapy: Current reality and future possibilities. Psychother Theory Res Pract Train 2003;40:55-67.

6. Proudfoot J, Goldberg D, Mann A, Everitt B, Marks I, Gray JA. Computerized, interactive, multimedia cognitive behavioural program for anxiety and depression in general practice. Psychol Med 2003;33:217-27.

7. White J, Jones R, McGarry E. Cognitive behavioural computer therapy for the anxiety disorders: A pilot study. J Mental Health UK 2000;9:505-16.

8. Dolezal-Wood S, Belar CD, Snibbe J. A comparison of computer assisted psychotherapy and cognitive behavioral therapy in groups. J Clin Psychol Med Settings 1998;5:103-15.

9. Kenardy J, Adams C. Computers in cognitive behaviour therapy. Aus Psychol 1993;28:189-94.
10. Proudfoot J, Swain S, Widmer S, et al. The development and beta test of a computer therapy program for anxiety and depression: Hurdles and lessons. Comput Hum Behav 2003;19:277-89.

11. Newman MG, Consoli AJ, Taylor CB. A palmtop computer program for the treatment of generalized anxiety disorder. Behav Modif 1999;23:597-619.

12. Newman MG, Consoli A, Taylor CB. Computers in assessment and cognitive behavioral treatment of clinical disorders: Anxiety as a case in point. Behav Therapy 1997;28:211-35.

13. Gruber K, Moran PJ, Roth WT, Taylor CB. Computer assisted cognitive behavioral group therapy for social phobia. Behav Therapy 2001;32:155-65.

14. Selmi PM, Klein MH, Greist JH, et al. Computer administered cognitive behavioral therapy for depression. Am J Psychiatry 1990;147:51-6.

15. Zabinski MF, Celio AA, Wilfley DE, Taylor CB. Prevention of eating disorders and obesity via the Internet. Cogn Behav Ther 2003;32:137-50.

16. Zabinski MF, Celio AA, Jacobs MJ, Manwaring J, Wilfley DE. Internet based prevention of eating disorders. Eur Eating Disorders Review 2003;11:183-97.

17. Zabinski MF, Pung MA, Wilfley DE, et al. Reducing risk factors for eating disorders: Targeting at risk women with a computerized psychoeducational program. Int J Eating Disorders 2001;29:401-8.

18. Riley S, Veale D. The internet and its relevance to cognitive behavioural psychotherapists. Behav Cogn Psychother 1999;27:37-46.

19. Gershon JR. Virtual reality as a distractor during an invasive medical procedure for pediatric cancer patients. Dissertation Abstracts International: Section B: The Sciences \& Engineering 2003;63(11B):5514.

20. Hoffman HG, Patterson DR, Magula J, et al. Water rriendly virtual reality pain control during wound care. J Clin Psychol 2004;60:189-95.

21. Patterson DR, Tininenko JR, Schmidt AE, Sharar SR. Virtual reality hypnosis: A case report. Int J Clin Exp Hypn 2004;52:27-38.

22. Gatchel RJ, Turk DC, eds. Psychological Approaches to Pain Management. New York: Guilford Publications, 1996.

23. Eccleston C, Morley S, Williams A, Yorke L, Mastroyannopoulou K. Systematic review of randomised controlled trials of psychological therapy for chronic pain in children and adolescents, with a subset meta analysis of pain relief. Pain 2002;99:157-65.

24. Raine R, Haines A, Sensky T, Hutchings A, Larkin K, Black N. Systematic review of mental health interventions for patients with common somatic symptoms: Can research evidence from secondary care be extrapolated to primary care? BMJ 2002;325:1082-5.

25. Hawkins RMF. A systematic meta review of hypnosis as an empirically supported treatment for pain. Pain Rev 2001;8:47-73.

26. Milling LS, Levine MR, Meunier SA. Hypnotic enhancement of cognitive behavioral interventions for pain: An analogue treatment study. Health Psychol 2003;22:406-13.

27. Montgomery GH, DuHamel KN, Redd WH. A meta analysis of hypnotically induced analgesia: How effective is hypnosis? Int J Clin Exp Hypn 2000;48:138-53

28. Grant CD, Nash MR. The Computer-Assisted Hypnosis Scale: Standardization and norming of a computer administered measure of hypnotic ability. Psychol Assess 1995;7:49-58.

29. Murthy BR, Pollack GM, Brouwer KL. Contribution of morphine-Gglucuronide to antinociception following intravenous administration of morphine to healthy volunteers. J Clin Pharmacol 2002;42:569-76.

30. Gintzler AR, Liu NJ. Ovarian sex steroids activate antinociceptive systems and reveal gender-specific mechanisms. In: Fillingim R, ed. Sex, Gender and Pain. Progress in Pain Research and Management. Seattle: IASP, 2000;17:89-108.

31. Hinkley B, Jaremko M. Addressing the psychosocial needs of orthopedic pain patients. Percept Mot Skills 1992;74:1137-8.

32. Stevens MJ. Interaction of coping style and cognitive strategies in the management of acute pain. Imagination, Cognition, and Personality 1991-1992;11:225-32. 


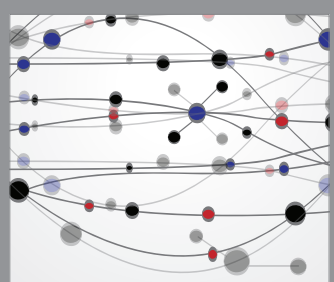

The Scientific World Journal
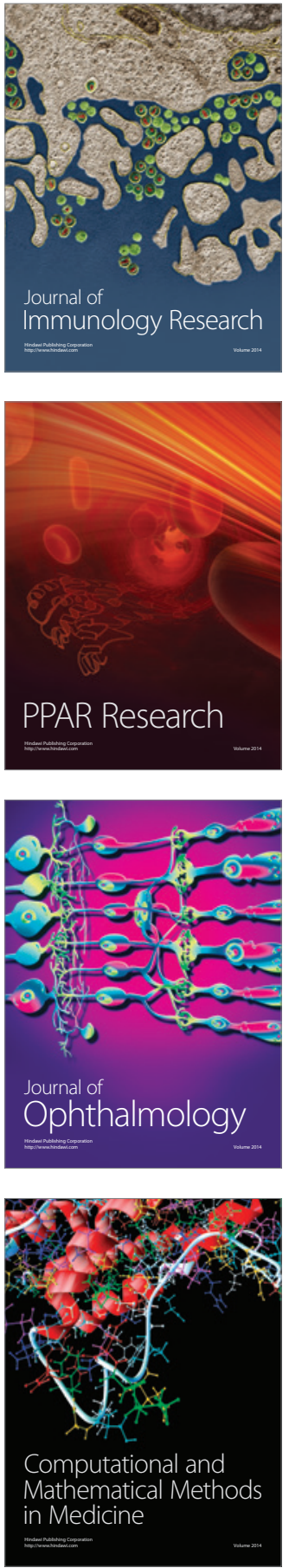

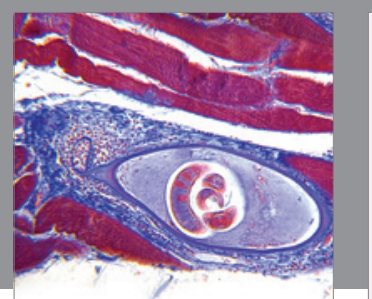

Gastroenterology Research and Practice

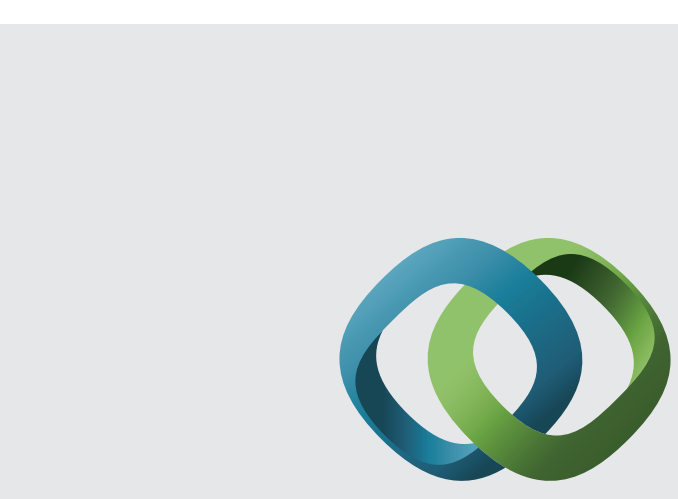

\section{Hindawi}

Submit your manuscripts at

http://www.hindawi.com
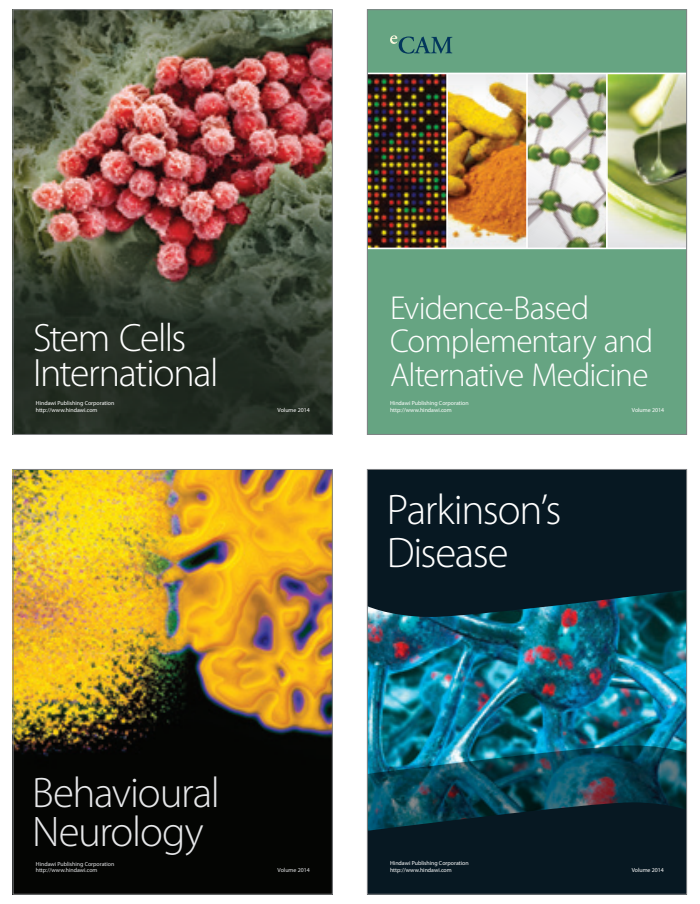
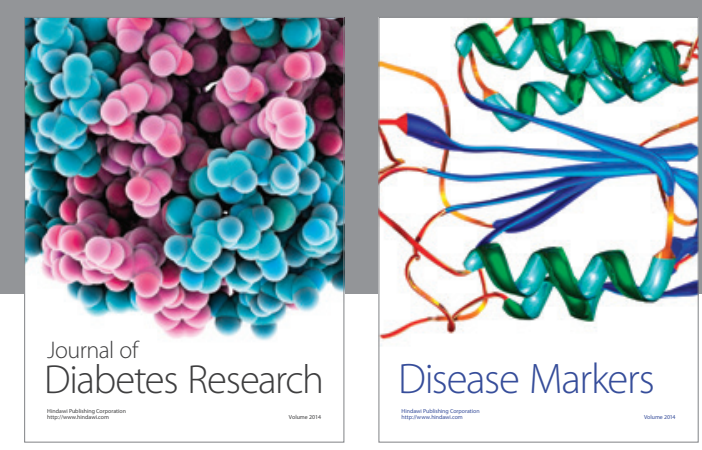

Disease Markers
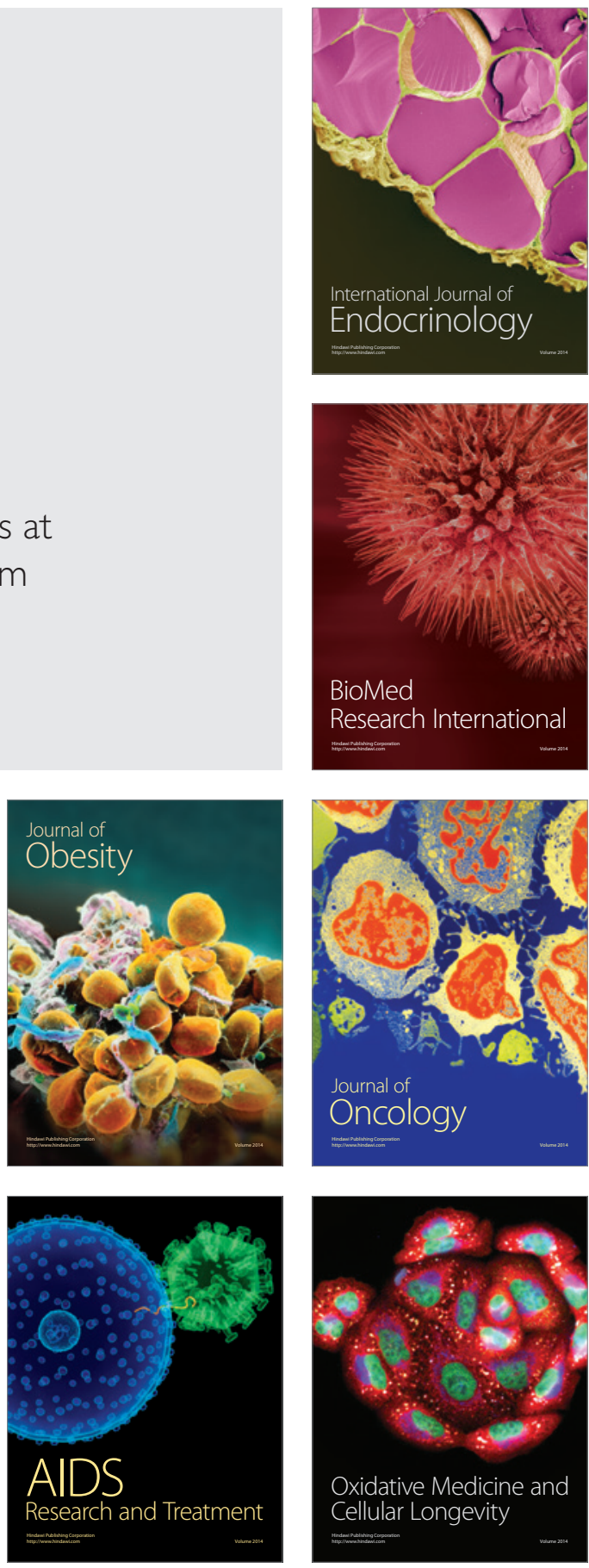